\title{
REGULARLY ORDERED GROUPS
}

PAUL F. CONRAD

1. Introduction. In [2] Robinson and Zakon present a metamathematical study of regularly ordered abelian groups, and in [3] Zakon proves the theorems that are stated without proofs in [2] and further develops the theory of these groups by algebraic methods. It is apparent from either paper that there is a close connection between regularly ordered groups and divisible ordered groups. In this note we establish this connection. For example, an ordered group $G$, with all convex subgroups normal, is regularly ordered if and only if $G / C$ is divisible for each nonzero convex subgroup $C$ of $G$. If $H$ is an ordered group with a convex subgroup $Q$ that covers 0 , then $H$ is regular if and only if $H / Q$ is divisible. Thus a regularly and discretely ordered abelian group is an extension of an infinite cyclic group by a rational vector space. In any case a regularly ordered group is "almost" divisible. In addition we use the notion of J-regularity, which is slightly weaker than regularity, and our results are not restricted to abelian groups.

Throughout this paper all groups, though not necessarily abelian, will be written additively, $R$ will denote the group of all real numbers with their natural order, and $G$ will denote a linearly ordered group (notation $o$-group). A subset $S$ of $G$ is convex if the relations $a<x<b$, $a, b \in S, x \in G$ always imply that $x \in S$. A subgroup of $G$ with this property is called a convex subgroup. $G$ is said to be regular if for every infinite convex subset $S$ of $G$ and every positive integer $n$, there exists an element $g$ in $G$ such that $n g \in S$. Clearly a divisible $o$-group is regular. If $a, b \in G$ and $a<b$, then $\{x \in G: a<x<b\}$ is the interval determined by $a$ and $b$, and we shall denote this interval by $(a<b)$. $G$ is said to be J-regular if for every infinite interval $(a<b)$ in $G$ and every positive integer $n$, there exists an element $g$ in $G$ such that $n g \in(a<b)$. It is clear that if $G$ is regular, then it is J-regular. Also, if $G$ is densely ordered and J-regular, then it is regular. For in this case every infinite convex subset of $G$ contains an infinite interval. In $\$ 4$ we give examples of abelian 0 -groups that are 3 -regular, but not regular.

Zakon shows (Theorem 2.4 in [3]) that an Archimedean o-group $A$ is regular and hence 5-regular. This is clear if $A$ is cyclic, and if $A$ is not cyclic, then (without loss of generality) $n A$ is dense in $R$ for all positive integers $n$, and so $A$ is regular.

Received by the editors August 18, 1961. 
2. The relationship between regularity, J-regularity and divisibility. Let $\Gamma$ be the set of all ordered pairs $\left(G^{\gamma}, G_{\gamma}\right)$ of convex subgroups of $G$ such that $G^{\gamma}$ covers $G_{\gamma}$. Each $G^{\gamma} / G_{\gamma}$ is an Archimedean o-group, and hence is $o$-isomorphic to a subgroup of $R$, and is regularly ordered. Define that $\left(G^{\alpha}, G_{\alpha}\right)>\left(G^{\beta}, G_{\beta}\right)$ if $G_{\alpha} \supseteq G^{\beta}$. This is a linear ordering of $\Gamma$, and the rank of $G$ is the order type of $\Gamma$. In particular, $G$ has rank 1 if and only if $G$ is Archimedean. In Propositions 1 through 4 and their corollaries let $C$ be a nonzero normal convex subgroup of $G$. It is easy to show that if $G$ is regular (J-regular), then $C$ is regular (J-regular) and if $G$ is J-regular, then $G / C$ is regular, but we do not need these results.

Proposition 1. If $G$ is regular, then $G / C$ is divisible. ${ }^{1}$

Proof. It suffices to show that $C+n G \supseteq G$ for all positive integers $n$. But for each $g$ in $G$, the set $C-g$ is infinite and convex and hence meets $n G$. Therefore $g \in C+n G$.

Proposition 2. If $G$ is J-regular, then $G / C$ is divisible except possibly when $C$ is discretely ordered of rank 1 , in which case $G / C$ is densely ordered.

Proof. If $C$ is densely ordered or the rank of $C$ exceeds 1 , then $C$ contains an infinite interval $(0<c)$. For each $g \in G$ and $n>0$ the interval $(g<g+c)$ is infinite and hence $g<n x<g+c$ for some $x$ in $G$. Therefore $C+g=C+n x=n(C+x)$, and it follows that $G / C$ is divisible.

Next assume that $C$ is discretely ordered and has rank 1 . If $G / C$ has no convex subgroup that covers 0 , then clearly $G / C$ is densely ordered. If $Q$ is the convex subgroup of $G / C$ that covers 0 , then $Q=C^{\prime} / C$, where $C^{\prime}$ is the convex subgroup of $G$ that covers $C$, and it suffices to show that $C^{\prime} / C$ is densely ordered. If $C^{\prime} / C$ is discretely ordered, then $C^{\prime}$ is $o$-isomorphic to a direct sum $I \oplus I$ of integers that is lexicographically ordered, say from the right $[1, \mathrm{p} .39]$. The interval $((0,1)<(0,2))$ is infinite and consists of the elements

$$
\{(n, 1) \text { and }(-n, 2): n>0\} \text {. }
$$

Clearly this interval contains no elements of the form $3(x, y)$, and since $C^{\prime}$ is convex in $G$ it follows (without loss of generality) that $((0,1)<(0,2))$ is an infinite interval in $G$ and that $((0,1)<(0,2))$ $\cap 3 G$ is the null set, but this contradicts the assumption that $G$ is J-regular. Therefore $C^{\prime} / C$ is densely ordered and so is $G / C$.

1 The author wishes to thank the referee for the proof of Proposition 1. 
Proposition 3. If every convex subgroup of $G$ is normal, and if $G$ satisfies both of the following conditions, then $G$ is J-regular.

(a) If $C$ has rank 1 , then $G / C$ is densely ordered.

(b) If the rank of $C$ exceeds 1 or if $C$ is densely ordered, then $G / C$ is divisible.

Proof. Let $(a<b)$ be an infinite interval in $G$, where $a \geqq 0$, and let $n$ be a positive integer. Let $C^{\prime}$ be the intersection of all convex subgroups of $G$ that contain $a-b$, and let $C$ be the join of all convex subgroups of $G$ that do not contain $a-b$. It follows that $C^{\prime}$ covers $C$, $a-b \in C^{\prime} \backslash C$ and $C+a<C+b$. If $C \neq 0$, then by (a) and (b) $C^{\prime} / C$ is densely ordered and by (b) $G / C^{\prime}$ is divisible. If $C=0$, then $(0<b-a)$ is an infinite interval in the Archimedean group $C^{\prime}$, and hence $C^{\prime}$ is densely ordered. Thus, by (b), $G / C^{\prime}$ is divisible. Therefore in any case $C^{\prime} / C$ is densely ordered and $n d \equiv a \equiv b \bmod C^{\prime}$ for some $d$ in $G$. Let $D=C+d, Y \in C^{\prime} / C$, and assume (without loss of generality) that $C^{\prime} / C$ is a subgroup of $R$. Then $D+Y-D=k Y$ for some $0<k \in R$, because all order preserving automorphisms of a subgroup of $R$ have this form, and by induction

$$
n(Y+D)=\left(k^{n-1}+k^{n-2}+\cdots+k+1\right) Y+n D .
$$

Note that $r=k^{n-1}+\cdots+1$ is a positive real number that depends only on $n$ and $D$, and that

$$
C+a<n(Y+D)<C+b
$$

if and only if

$$
C+a-n D<r Y<C+b-n D \text {. }
$$

Since $C^{\prime} / C$ is densely ordered, it is dense in $R$, but this means that $r\left(C^{\prime} / C\right)$ is dense in $R$, and hence $r\left(C^{\prime} / C\right)$ is dense in $C^{\prime} / C$. It follows that there exists an element $Y$ in $C^{\prime} / C$ that satisfies (2) and hence (1). $n(Y+D)=n\left(C+d^{\prime}\right)=C+n d^{\prime}$, where $d^{\prime} \in G$, and hence $a<n d^{\prime}<b$. Therefore $G$ is $J$-regular.

CoROllaRy. Let $Q$ and $Q^{\prime}$ be convex subgroups of the discretely ordered group $G$ such that $Q$ covers 0 and $Q^{\prime}$ covers $Q$. If $G / Q$ is densely ordered and $G / Q^{\prime}$ is divisible, then $G$ is J-regular.

Proof. Since inner automorphisms preserve order, $Q$ and $Q^{\prime}$ are normal. Let $(a<b)$ be an infinite interval in $G$, with $a \geqq 0$, and let $n$ be a positive integer. If $Q^{\prime}+a<Q^{\prime}+b$, then since $G / Q^{\prime}$ is divisible, $Q^{\prime}+a<Q^{\prime}+n g<Q^{\prime}+b$ for some $g \in G$, and hence $n g \in(a<b)$. If $a-b \in Q$, then $(0<b-a)$ is an infinite interval in the cyclic group $Q$, 
which is impossible. Finally, if $a-b \in Q^{\prime} \backslash Q$, then by the argument in the proof of Proposition 3 (where $C^{\prime}=Q^{\prime}$ and $C=Q$ ), $a<n d^{\prime}<b$ for some $d^{\prime} \in G$. Therefore $G$ is 3-regular.

Proposition 4. If every convex subgroup of $G$ is normal, and if $G / C$ is divisible for all nonzero convex subgroups $C$ of $G$, then $G$ is regular.

Proof. Let $S$ be an infinite convex subset of $G$, and let $n$ be a positive integer. We must show that $n g \in S$ for some $g$ in $G$. This follows from Proposition 3 if there exists an infinite interval in $S$. Suppose that all intervals $(a<b)$ with $a, b \in S$ are finite. In particular, $G$ is discretely ordered. Let $C$ be the cyclic convex subgroup of $G$ that covers 0 and let $c$ be the positive generator of $C$. An inner automorphism of $G$ preserves order and hence must induce the identity automorphism on $C$. Therefore $C$ is in the center of $G$. If $a, b \in S$ and $a<b$, then $a \equiv b \bmod C$. For otherwise $C+a<C+b$ in the divisible group $G / C$, and it follows that $(a<b)$ is an infinite interval, a contradiction. Thus we have that $S=C^{*}+s$, where $s$ is a fixed element in $S$ and $C^{*}$ is an infinite convex subset of $C$. Since $G / C$ is divisible, $n d \equiv s \bmod C$ for some $d$ in $G$. Thus $n d=q c+s$ for some integer $q$, and it is clear that $(n t+q) c \in C^{*}$ for some integer $t$. Therefore $n(t c+d)=n t c+n d$ $=n t c+q c+s=(n t+q) c+s \in S$.

Corollary. If $Q$ is a convex subgroup of $G$ that covers 0 , and if $G / Q$ is divisible then $G$ is regular.

Proof. Let $S$ be an infinite convex subset of positive elements in $G$, and let $n$ be a positive integer. If $S$ contains no infinite interval, then, by the proof of Proposition 4, ng $\in S$ for some $g \in G$. Suppose that $(a<b)$ is an infinite interval in $S$. If $Q+a<Q+b$, then $Q+a<Q$ $+n g<Q+b$ for some $g \in G$ because $G / Q$ is divisible. Thus $n g \in(a<b)$. If $Q+a=Q+b$, then $n d \equiv a \equiv b \bmod Q$ for some $d \in G$ and by the proof of Proposition 3 (where $C^{\prime}=Q$ and $C=0$ ), there exists an element $y$ in $Q$ such that $a<n(y+d)<b$.

3. The main theorems. The following two theorems are immediate consequences of the propositions in the last section and their corollaries.

THEOREM I. Suppose that every convex subgroup of the o-group $G$ is normal.

(a) $G$ is regular if and only if $G / C$ is divisible for every nonzero convex subgroup $C$ of $G$. In particular, if $G \neq 0$ is abelian and regular, then $G$ is divisible if and only if it contains a nonzero divisible convex subgroup. 
Suppose, in addition, that $G$ is discretely ordered and let $Q$ be the convex subgroup of $G$ that covers 0 .

(b) $G$ is J-regular if and only if $G / Q$ is densely ordered and $G / C$ is divisible for every convex subgroup $C$ of $G$ that properly contains $Q$.

TheOREM II. Suppose that $G$ is an o-group with a convex subgroup $Q$ that covers 0 .

(a) $G$ is regular if and only if $G / Q$ is divisible. In particular, if $G$ is regular and a central extension of $Q$, then $G$ is divisible if and only if $Q$ is divisible. Suppose, in addition, that $G$ is discretely ordered ( $\equiv Q$ is discretely ordered) and that $Q^{\prime}$ is a convex subgroup of $G$ that covers $Q$.

(b) $G$ is J-regular if and only if $G / Q$ is densely ordered and $G / Q^{\prime}$ is divisible.

Note that Theorem II characterizes regular and J-regular groups of finite rank. Also, a regular discretely ordered abelian group is an extension of an infinite cyclic group by a rational vector space.

4. Examples and remarks. Let $A$ be the direct sum of the groups $D$ of rational numbers and the integral multiples of $\pi$ with the natural order. Let $I$ be the group of integers, and let $G=I \oplus A$ lexicographically ordered from the right. Thus $G / I$ is densely ordered, but not divisible. By Theorem II, $G$ is J-regular but not regular.

For each $m=-1,-2, \cdots$, let $D_{m}$ be the group of rationals. Let $A$ be the large direct sum $\cdots \oplus D_{-2} \oplus D_{-1}$, lexicographically ordered from the right, and let $B$ be the subgroup of $A$ that is generated by the small direct sum of the $D_{m}$ and the "long integral constants"

$$
(\cdots, n, n, n)
$$

where $n$ is an integer. Let $G=I \oplus B$ lexicographically ordered from the right. $G / I$ is not divisible, and hence by Theorem II, $G$ is not regular, but $G / I$ is densely ordered and $G / C$ is divisible for all convex subgroups $C$ of $G$ that properly contain $I$. Thus, by Theorem I, $G$ is J-regular. Note that $B$ is regular, and densely ordered, but not divisible.

We next give an example of an $o$-group $G$ such that $G / C$ is divisible for all nonzero convex normal subgroups $C$ of $G$, but $G$ is not 3-regular. This helps to justify the hypothesis in Theorem I that all convex subgroups are normal. Let $G$ be the wreath product of the integers $I$ by the rationals $D$. Then $G$ is a splitting extension of the small direct sum $S$ of $D$ copies of the integers by $D$. If we order $G$ lexicographically, then $S$ is the only proper convex normal subgroup of $G$, and $G / S$ is divisible, because it is isomorphic to $D$. If $C$ is any convex 
subgroup of $S$, then $S / C$ is not divisible, hence $S$ is not regular, and thus $G$ is not regular. Since $G$ is densely ordered, $G$ is also not 5 regular.

Let us call an 0 -group $G \mathfrak{N}$-regular if for every nonempty interval $(a<b)$ in $G$ and every positive integer $n$, there exists an element $g$ in $G$ such that $n g \in(a<b)$. Clearly if $G$ is $\Re$-regular, then it is densely ordered, and hence each nonempty interval is infinite. Therefore $G$ is $\mathfrak{T}$-regular if and only if $G$ is regular and densely ordered.

Let $A$ be an abelian $o$-group with a convex subgroup $Q$ that covers 0 . For each positive integer $n$, the mapping $\alpha_{n}$ of $n Q+q$ upon $n A+q$ is an isomorphism of $Q / n Q$ into $A / n A$. The following are equivalent:

(i) $A$ is regular,

(ii) $A / Q$ is divisible,

(iii) $\alpha_{n}$ is an isomorphism of $Q / n Q$ onto $A / n A$ for each $n>0$.

Proof. (i) and (ii) are equivalent by Theorem II. Clearly (iii) is equivalent to $a \equiv x \bmod n A$ having a solution in $Q$ for each $a \in A$ and each $n>0$. Consider $a \in A$ and $n>0$. If (iii) is satisfied, then $a=q+n a^{\prime}$ for some $q \in Q$ and $a^{\prime} \in A$, and hence $Q+a=Q+n a^{\prime}=n\left(Q+a^{\prime}\right)$. Thus $A / Q$ is divisible. Conversely, if $A / Q$ is divisible, then $Q+a=Q+n a^{\prime}$ for some $a^{\prime} \in A$, hence $a \equiv x \bmod n A$ has a solution in $Q$. Thus (iii) is satisfied.

In particular, if $A$ is discretely ordered, then $Q / n Q$ is a cyclic group of order $n$. Thus a discretely ordered abelian group $A$ is regular if and only if $A / n A$ is of order $n$ for all $n>0$. This is Zakon's Theorem 2.1. Similarly other results of Zakon can be proven using Theorems I and II. For example, suppose that $A_{0}$ and $A$ are regular discretely ordered abelian groups and that $Q \subseteq A_{0} \subseteq A$, where $Q$ is the convex subgroup of $A$ that covers 0 . Then, by Theorem II, $A / Q$ and $A_{0} / Q$ are divisible (and torsion free), hence $A / Q=A_{0} / Q \oplus D$, where $D$ is divisible and torsion free. Thus $D \cong(A / Q) /\left(A_{0} / Q\right) \cong A / A_{0}$ is divisible and torsion free. Therefore $A_{0}$ is pure and basic in $A$. This is Zakon's Corollary 1.2 .

\section{REFERENCES}

1. P. Conrad, Non-abelian ordered groups, Pacific J. Math. 9 (1959), 25-41.

2. A. Robinson and E. Zakon, Elementary properties of ordered abelian groups, Trans. Amer. Math. Soc. 96 (1960), 222-236.

3. E. Zakon, Generalized Archimedean groups, Trans. Amer. Math. Soc. 99 (1961), 21-40.

TUlane UNIVERSITY 